\title{
Outage Minimization For State Estimation Using Multiple Sensors
}

\author{
Alex S. Leong, Subhrakanti Dey, Girish N. Nair* \\ Priyank Sharma** \\ * Department of Electrical and Electronic Engineering, University of \\ Melbourne, Parkville, Vic. 3010, Australia (e-mail: \{asleong, sdey, \\ gnair\}@unimelb.edu.au) \\ ** Department of Electrical Engineering, Indian Institute of \\ Technology, Kanpur, India (e-mail: priyanks@iitk.ac.in)
}

\begin{abstract}
This paper studies the outage minimization problem for state estimation of a scalar linear dynamical system using multiple sensors. The sensors amplify and forward their measurements to a remote fusion center over wireless fading channels. For stable systems, the resulting infinite horizon problem is a constrained Markov decision process (MDP) that can be solved using dynamic programming techniques. A suboptimal power allocation that is less computationally intensive is also proposed, and numerical results demonstrate very close performance to the power allocation obtained from the solution of the MDP. For unstable systems, a finite horizon formulation of the outage minization problem is also presented.
\end{abstract}

Keywords: Outage probability, sensor networks, state estimation

\section{INTRODUCTION}

In real time applications notions of outage are often used to quantify the time periods when the performance of a system is below what is desired. For instance, in mobile telephony outages could correspond to times where the audio quality is very poor, and in tracking applications outages might correspond to instances where the location of a target cannot be determined to a desired accuracy.

In communications and information theory the notion of delay-limited or zero-outage capacity was introduced in Hanly and Tse (1998). The concepts of information outage probability and outage capacity, and the optimal power allocation to minimize the outage probability subject to an average power constraint, was subsequently studied in Caire et al. (1999). Extensions of the outage concept in communications theory include the delay constrained outage capacity problem in Negi and Cioffi (2002) and the notion of service outage in Luo et al. (2005). In the signal processing literature, the notion of estimation outage for the distributed estimation of an i.i.d. source was introduced in Cui et al. (2007).

In much of these previous works, the systems that have been studied have been memoryless, so that how the resources are allocated at one time instant does not necessarily affect the evolution of the system at future times. The focus of this paper is on extending the notions of estimation outage, and solving the outage minimization problem, for dynamical systems. In particular we consider state estimation of a scalar linear dynamical system using multiple sensors, where the sensors transmit their measurements to a fusion center over wireless channels using the analog amplify and forward technique of Gastpar and

^ This work was supported by the Australian Research Council.
Vetterli (2003), which is a scheme that has been shown to be asymptotically optimal in certain distributed estimation scenarios. An outage will be defined as the event that the estimation error covariance exceeds a given threshold, and we are interested in how to optimally allocate the transmit powers of the sensors in order to minimize the probability of outage, subject to an average sum power constraint. In the case of stable systems, we formulate the problem over an infinite horizon. This will turn out to be a constrained Markov decision process (MDP), which we can transform using a Lagrangian technique into an unconstrained MDP, that can then be solved with standard dynamic programming algorithms. In the case of unstable systems, an infinite horizon problem formulation is not appropriate since increasingly large amounts of power will need to be transmitted, thus we use a finite horizon formulation instead. Dynamic programming techniques have also been used in solving related problems such as the delay constrained outage capacity problem in Negi and Cioffi (2002), and estimation error minimization problems for hidden Markov model state estimation in Huang and Dey (2006) and Ghasemi and Dey (2008).

The organization of the paper is as follows. Section 2 presents our system model and statement of the outage minimization problem for stable systems. Section 3 derives some conditions on the distortion threshold that affect the solvability of the problem. The outage minimization problem is solved in Section 4, and a sub-optimal policy is proposed in Section 5. Numerical results are presented in Section 6. Unstable systems are considered in Section 7.

\section{SYSTEM MODEL}

We consider a discrete time scalar linear system given by

$$
x_{k+1}=a x_{k}+w_{k}
$$


where $x_{k}, w_{k}, a \in \mathbb{R}$, and $w_{k}$ is white Gaussian noise ${ }^{1}$ with zero mean and variance $\sigma_{w}^{2}$.

The system is observed by $M$ different sensors with observations

$$
y_{i, k}=c_{i} x_{k}+v_{i, k}, i=1, \ldots, M
$$

with $y_{i, k}, v_{i, k}, c_{i} \in \mathbb{R}$, and $v_{i, k}$ is white Gaussian noise with zero mean and variance $\sigma_{i}^{2}$.

The sensors then send their measurements over wireless channels to a fusion centre. We assume that the sensors use the analog amplify and forward technique of Gastpar and Vetterli (2003), where the sensor transmitter amplifies $y_{i, k}$ by a factor $\alpha_{i, k}$ and sends it to the fusion centre over a fading channel. The different fading channels are taken to be orthogonal, as in Cui et al. (2007). We remark that a non-orthogonal multi-access transmission scheme can also be considered, see Gastpar and Vetterli (2003), but for simplicity we will restrict ourselves to the orthogonal scheme in this paper. The received signals at the fusion centre can be written as

$$
\begin{aligned}
z_{i, k} & =\alpha_{i, k} \sqrt{g_{i, k}} y_{i, k}+n_{i, k} \\
& =\alpha_{i, k} \sqrt{g_{i, k}} c_{i} x_{k}+\alpha_{i, k} \sqrt{g_{i, k}} v_{k}+n_{i, k}, i=1, \ldots, M
\end{aligned}
$$

where $g_{i, k} \geq 0$ are the random channel gains which are assumed to be known (i.e. we have channel state information), $n_{i, k}$ is white Gaussian with zero mean and variance $\sigma_{n}^{2}$, and $\alpha_{i, k}$ are the amplification factors in the analog forwarding scheme. We assume a block fading model, with the channel gains $g_{i, k}$ being i.i.d. over time. We also assume that $x_{0}, w_{k}, v_{i, k}, g_{i, k}$ and $n_{i, k}$ are mutually independent.

Call $z_{k}=\left(z_{1, k}, \ldots, z_{M, k}\right)^{T}, g_{k}=\left(g_{1, k}, \ldots, g_{M, k}\right)^{T}, \bar{C}_{k}=$ $\left(\alpha_{1, k} \sqrt{g_{1, k}} c_{1}, \ldots, \alpha_{M, k} \sqrt{g_{M, k}} c_{M}\right)^{T}, \bar{v}_{k}=\left(\alpha_{1, k} \sqrt{g_{1, k}} v_{1, k}+\right.$ $\left.n_{1, k}, \ldots, \alpha_{M, k} \sqrt{g_{M, k}} v_{M, k}+n_{M, k}\right)^{T}, \bar{R}_{k}=\operatorname{diag}\left(\alpha_{1, k}^{2} g_{1, k} \sigma_{1}^{2}+\right.$ $\left.\sigma_{n}^{2}, \ldots, \alpha_{M, k}^{2} g_{M, k} \sigma_{M}^{2}+\sigma_{n}^{2}\right)$. The equations in (2) can then be written as

$$
z_{k}=\bar{C}_{k} x_{k}+\bar{v}_{k}
$$

where $\bar{v}_{k}$ has the time-varying covariance matrix $\bar{R}_{k}$. The equations (1) and (3) form a linear time-varying system whose state $x_{k}$ can be optimally estimated by a timevarying Kalman filter at the fusion centre. Define the state estimate and error covariance as

$$
\begin{aligned}
\hat{x}_{k+1 \mid k} & =\mathbb{E}\left[x_{k+1} \mid z_{1}, \ldots, z_{k}, g_{1}, \ldots, g_{k}\right] \\
P_{k+1 \mid k} & =\mathbb{E}\left[\left(x_{k+1}-\hat{x}_{k+1 \mid k}\right)^{2} \mid z_{1}, \ldots, z_{k}, g_{1}, \ldots, g_{k}\right]
\end{aligned}
$$

In the following, we will also use the short hand notation $P_{k+1}=P_{k+1 \mid k}$.

One can then show from the time-varing Kalman filter equations and an application of the matrix inversion lemma that the recursion for the error covariance satisfies:

$$
\begin{aligned}
P_{k+1} & =a^{2} P_{k}-a^{2} P_{k}^{2} \bar{C}_{k}^{T}\left(\bar{C}_{k} P_{k} \bar{C}_{k}^{T}+\bar{R}_{k}\right)^{-1} \bar{C}_{k}+\sigma_{w}^{2} \\
& =\frac{a^{2} P_{k}}{1+P_{k} \sum_{i=1}^{M} \frac{\alpha_{i, k}^{2} g_{i, k} c_{i}^{2}}{\alpha_{i, k}^{2} g_{i, k} \sigma_{i}^{2}+\sigma_{n}^{2}}}+\sigma_{w}^{2}
\end{aligned}
$$

\footnotetext{
1 We call $\left\{w_{k}\right\}$ white if $w_{k}$ and $w_{l}$ are independent for $k \neq l$.
}

The sensor transmit power $\gamma_{i, k}$ used by the $i$-th sensor in transmitting its measurement to the fusion centre at time $k$ is defined as

$$
\gamma_{i, k}=\alpha_{i, k}^{2} \mathbb{E}\left[y_{i, k}^{2}\right]=\alpha_{i, k}^{2}\left(c_{i}^{2} \mathbb{E}\left[x_{k}^{2}\right]+\sigma_{i}^{2}\right)
$$

\subsection{Problem statement}

We will consider here stable linear systems, i.e. $|a|<1$ (see Section 7 for the case of unstable systems). Then $\mathbb{E}\left[x_{k}^{2}\right]=\sigma_{w}^{2} /\left(1-a^{2}\right)$ and (5) simplifies to

$$
\gamma_{i, k}=\alpha_{i, k}^{2}\left(c_{i}^{2} \frac{\sigma_{w}^{2}}{1-a^{2}}+\sigma_{i}^{2}\right)
$$

Let us call $\alpha_{k}=\left(\alpha_{1, k}, \ldots, \alpha_{M, k}\right)^{T}, \gamma_{k}=\left(\gamma_{1, k}, \ldots, \gamma_{M, k}\right)^{T}$. The problem we consider in this paper is to choose the $\alpha_{k}$ 's (and hence the $\gamma_{k}$ 's) to minimize the outage probability subject to an average power constraint $\mathcal{P}$ on the sum of the transmitted powers. Here, we declare an outage event if the error covariance $P_{k+1}$ exceeds some distortion threshold $D$. More formally, we want to solve over an infinite horizon the problem:

$$
\begin{aligned}
& \min _{\left\{\gamma_{k}\right\}} \limsup _{K \rightarrow \infty} \frac{1}{K} \sum_{k=0}^{K-1} \operatorname{Pr}\left(P_{k+1}>D\right) \\
& =\min _{\left\{\gamma_{k}\right\}} \limsup _{K \rightarrow \infty} \frac{1}{K} \sum_{k=0}^{K-1} \mathbb{E}\left[1\left(P_{k+1}>D\right)\right] \\
& \text { s.t. } \limsup _{K \rightarrow \infty} \frac{1}{K} \sum_{k=0}^{K-1} \mathbb{E}\left[\sum_{i=1}^{M} \gamma_{i, k}\right] \leq \mathcal{P}
\end{aligned}
$$

where $1(\cdot)$ is the indicator function.

\section{CONDITIONS ON $D$}

In this section we will derive some conditions on the threshold $D$ which will affect the solvability of problem (6).

For an initial simple bound, note that for stable systems the following holds:

$$
\sigma_{w}^{2}<P_{k} \leq \frac{\sigma_{w}^{2}}{1-a^{2}}, \forall k
$$

Hence if $D \leq \sigma_{w}^{2}$, then $P_{k+1}$ will always exceed $D$, and if $D>\sigma_{w}^{2} /\left(1-a^{2}\right)$ then we will never have any outage events. Also note from (4) that given $P_{k}$, the error covariance at the next time instant $P_{k+1}$ is bounded by

$$
\frac{a^{2} P_{k}}{1+P_{k} \sum_{i=1}^{M} c_{i}^{2} / \sigma_{i}^{2}}+\sigma_{w}^{2}<P_{k+1} \leq a^{2} P_{k}+\sigma_{w}^{2} .
$$

Below we present some more precise conditions. In particular we will partition the range of $D$ such that given $P_{k}$, the condition $P_{k+1} \leq D$ can either: i) always be achieved, ii) never be achieved, or iii) can be achieved only for $P_{k}$ sufficiently small.

1) Suppose that at time $k, P_{k}=\sigma_{w}^{2} /\left(1-a^{2}\right)$. Then

$$
\begin{aligned}
\frac{a^{2} P_{k}}{1+P_{k} \sum_{i=1}^{M} c_{i}^{2} / \sigma_{i}^{2}}+\sigma_{w}^{2} & =\frac{a^{2} \sigma_{w}^{2}}{1-a^{2}+\sigma_{w}^{2} \sum_{i=1}^{M} c_{i}^{2} / \sigma_{i}^{2}}+\sigma_{w}^{2} \\
& \equiv D_{1}
\end{aligned}
$$

Hence we have the condition that if $D \geq D_{1}$, then $P_{k+1} \leq$ $D$ can be achieved in one time step for all $P_{k} \geq D$. 
2) Consider the values of $P_{k}$ such that

$$
\frac{a^{2} P_{k}}{1+P_{k} \sum_{i=1}^{M} c_{i}^{2} / \sigma_{i}^{2}}+\sigma_{w}^{2}>P_{k} .
$$

This can be easily shown to be equivalent to

$$
P_{k}<\frac{-B+\sqrt{B^{2}+4 \sigma_{w}^{2} \sum_{i=1}^{M} c_{i}^{2} / \sigma_{i}^{2}}}{2 \sum_{i=1}^{M} c_{i}^{2} / \sigma_{i}^{2}} \equiv D_{2},
$$

with $B=\left(1-a^{2}-\sigma_{w}^{2} \sum_{i=1}^{M} c_{i}^{2} / \sigma_{i}^{2}\right)$. Hence we now have the condition that if $D \leq D_{2}$ and $P_{k} \geq D$, then $P_{k+1} \leq D$ cannot be achieved (in one time step and therefore cannot be achieved in all subsequent time steps). This is a tight form of the condition $D \leq \sigma_{w}^{2}$ always resulting in outage mentioned at the start of this section.

3) In the case where $D$ satisfies $D_{2}<D<D_{1}$, we have the situation where given $P_{k}$, the condition $P_{k+1} \leq D$ can only be achieved for $P_{k}$ sufficiently small, more precisely when

or

$$
\frac{a^{2} P_{k}}{1+P_{k} \sum_{i=1}^{M} c_{i}^{2} / \sigma_{i}^{2}}+\sigma_{w}^{2}<D
$$

$$
P_{k}<\frac{D-\sigma_{w}^{2}}{a^{2}-\left(D-\sigma_{w}^{2}\right) \sum_{i=1}^{M} c_{i}^{2} / \sigma_{i}^{2}} .
$$

If this is not the case, then it will require more than one time step to bring the error covariance below the distortion threshold $D$. This has implications in that one cannot directly use the analogue of a scheme considered in Caire et al. (1999) as a suboptimal policy, see Section 5.

\section{MDP SOLUTION}

Problem (6) can be regarded as a constrained average cost MDP with $\left(P_{k}, g_{k}\right)=\left(P_{k}, g_{1, k}, \ldots, g_{M, k}\right)$ as the composite "state" and $\gamma_{k}=\left(\gamma_{1, k}, \ldots, \gamma_{M, k}\right)$ as the "action". In order to obtain non-trivial solutions to (6), we assume that the condition $D_{2}<D \leq \sigma_{w}^{2} /\left(1-a^{2}\right)$ of Section 3 holds for $D$. We will solve (6) using a similar approach to Ghasemi and Dey (2008), by converting the constrained MDP into an unconstrained MDP. We first introduce the Lagrangian:

$L^{\beta}=\limsup _{K \rightarrow \infty} \frac{1}{K}\left\{\sum_{k=0}^{K-1} \mathbb{E}\left[1\left(P_{k+1}>D\right)\right]+\beta \sum_{k=0}^{K-1} \mathbb{E}\left[\sum_{i=1}^{M} \gamma_{i, k}\right]\right\}$ where $\beta \geq 0$ is a weighting parameter that takes on the role of a Lagrange multiplier, and specifies the trade-off between the relative importance of total transmit power and outage probability. Note that from (4), $P_{k+1}$ is a function of $P_{k}, g_{k}, \gamma_{k}$, while $\gamma_{k}$ is assumed to be a function of $P_{k}$ and $g_{k}$. We then have the unconstrained problem

$$
\min _{\left\{\gamma_{k}\right\}} \limsup _{K \rightarrow \infty} \frac{1}{K} \sum_{k=0}^{K-1} \mathbb{E}\left[l^{\beta}\left(P_{k}, g_{k}, \gamma_{k}\right) \mid P_{0}, g_{0}\right]
$$

where $l^{\beta}\left(P_{k}, g_{k}, \gamma_{k}\right) \equiv 1\left(P_{k+1}>D\right)+\beta \sum_{i=1}^{M} \gamma_{i, k}$. In order to obtain numerical solutions to $(8)$ we will need to discretize the range of the quantities $P_{k}, g_{k}=\left(g_{1, k}, \ldots, g_{M, k}\right)$ and $\gamma_{k}=\left(\gamma_{1, k}, \ldots, \gamma_{M, k}\right)$. Let $P_{k}^{d}, g_{k}^{d}=\left(g_{1, k}^{d}, \ldots, g_{M, k}^{d}\right)$, and $\gamma_{k}^{d}=\left(\gamma_{1, k}^{d}, \ldots, \gamma_{M, k}^{d}\right)$ be the discretized versions of $P_{k}$, $g_{k}, \gamma_{k}$ respectively. One then has the following problem (9), the solution of which will approximate the solution to (8):

$$
\min _{\left\{\gamma_{k}^{d}\right\}} \limsup _{K \rightarrow \infty} \frac{1}{K} \sum_{k=0}^{K-1} \mathbb{E}\left[l^{\beta}\left(P_{k}^{d}, g_{k}^{d}, \gamma_{k}^{d}\right) \mid P_{0}^{d}, g_{0}^{d}\right]
$$

The Bellman equation associated with problem (9) can then be written as follows, with $\lambda$ representing the optimal average cost per stage, and $h$ the differential cost vector:

$$
\begin{aligned}
& \lambda+ h\left(P_{k}^{d}, g_{k}^{d}\right) \\
&= \min _{\gamma_{k}^{d}}\left[l^{\beta}\left(P_{k}^{d}, g_{k}^{d}, \gamma_{k}^{d}\right)\right. \\
&\left.\quad+\sum_{g_{k+1}^{d}, P_{k+1}^{d}} p\left(P_{k+1}^{r n d}, g_{k+1}^{d} \mid P_{k}^{d}, g_{k}^{d}, \gamma_{k}^{d}\right) h\left(P_{k+1}^{r n d}, g_{k+1}^{d}\right)\right] \\
&= \min _{\gamma_{k}^{d}}\left[l^{\beta}\left(P_{k}^{d}, g_{k}^{d}, \gamma_{k}^{d}\right)\right. \\
&\left.\quad+\sum_{g_{k+1}^{d}, P_{k+1}^{d}} p\left(g_{k+1}^{d}\right) p\left(P_{k+1}^{r n d} \mid P_{k}^{d}, g_{k}^{d}, \gamma_{k}^{d}\right) h\left(P_{k+1}^{r n d}, g_{k+1}^{d}\right)\right] \\
&=\min _{\gamma_{k}^{d}}\left[l^{\beta}\left(P_{k}^{d}, g_{k}^{d}, \gamma_{k}^{d}\right)+\sum_{g_{k+1}^{d}} p\left(g_{k+1}^{d}\right) h\left(P_{k+1}^{r n d}, g_{k+1}^{d}\right)\right]
\end{aligned}
$$

where $P_{k+1}^{r n d}$ is the value of $P_{k+1}$ (given $P_{k}^{d}, g_{k}^{d}, \gamma_{k}^{d}$ ) rounded to the nearest discretized value, such as in Huang and Dey (2006). The last line of (10) holds because $P_{k+1}^{r n d}$ is a deterministic function of $P_{k}^{d}, g_{k}^{d}, \gamma_{k}^{d}$, so that $p\left(P_{k+1}^{r n d} \mid P_{k}^{d}, g_{k}^{d}, \gamma_{k}^{d}\right)$ is either 0 or 1 .

Now given any two error covariances $\Sigma_{1}$ and $\Sigma_{2}$ satisfying $D_{2} \leq \Sigma_{1} \leq \sigma_{w}^{2} /\left(1-a^{2}\right)$ and $D_{2} \leq \Sigma_{2} \leq \sigma_{w}^{2} /\left(1-a^{2}\right)$, one can easily construct policies that can take $\Sigma_{1}$ to $\Sigma_{2}$ in a finite number of time steps. We may then use standard results from e.g. Bertsekas (2000) to conclude the existence of solutions to the Bellman equation (10). In this paper we will obtain solutions to the Bellman equation (10) numerically by using the relative value iteration algorithm, see e.g. Bertsekas (2000) and Puterman (1994).

\section{SUBOPTIMAL POLICIES}

The MDP approach of Section 4 is computationally demanding, particularly as the number of sensors increases since the dimensions of $g_{k}$ and $\gamma_{k}$ will increase with each extra sensor. In this section we will consider a simpler power allocation policy, that can be easily implemented even for large numbers of sensors, and whose performance is very close to that obtained from solving the MDP.

The motivation for our suboptimal policy comes from the solution of the outage minimization problem from communications theory studied in Caire et al. (1999). There, an outage is defined as the event that

$$
I_{M}\left(g_{k}, \gamma_{k}\right) \equiv \frac{1}{M} \sum_{i=1}^{M} \log \left(1+g_{i, k} \gamma_{i, k}\right)<R
$$

for some rate $R$, and where $I_{M}\left(g_{k}, \gamma_{k}\right)$ is defined as the instantaneous mutual information. The $M$ in (11) refers to the number of different blocks of an $M$-block fading channel, rather than different sensors, though the analogies with our situation are apparent. The index $k$ here is used to denote a frame of $M$ blocks.

The problem considered in Caire et al. (1999) is then to allocate the power over the $M$ blocks to minimize the outage probability subject to an average power constraint, i.e. 


$$
\begin{array}{r}
\min \operatorname{Pr}\left(I_{M}\left(g_{k}, \gamma_{k}\right)\right)<R \\
\text { s.t. } \mathbb{E}\left[\frac{1}{M} \sum_{i=1}^{M} \gamma_{i, k}\right] \leq \mathcal{P}
\end{array}
$$

The solution to this problem involves solving a subproblem:

$$
\begin{aligned}
& \min \frac{1}{M} \sum_{i=1}^{M} \gamma_{i, k} \\
& \text { s.t. } I_{M}\left(g_{k}, \gamma_{k}\right)=R
\end{aligned}
$$

that minimizes the power usage over the $M$ blocks $\frac{1}{M} \sum_{i=1}^{M} \gamma_{i, k}$, subject to the constraint $I_{M}\left(g_{k}, \gamma_{k}\right)=R$. If this minimizing sum power is less than a power threshold $s^{*}$, then the optimal power allocation is as given by the solution to the sub-problem (12). On the other hand, if the sum power required to solve the sub-problem exceeds the threshold $s^{*}$, then the optimal allocation is for transmission to be turned off. The threshold $s^{*}$ is the one that will satisfy the average sum power constraint, and can be determined either analytically in simple cases or via Monte Carlo simulations.

Motivated by this solution, the simple power allocation policy we propose for problem (6) is the following: Given $P_{k}$ and $g_{k}$, solve the sub-problem that minimizes the sum power subject to the constraint $P_{k+1}=D$. If the required sum power is less than a power threshold $s^{*}$, use this power allocation, otherwise don't transmit. The threshold $s^{*}$ is again determined by the average sum power constraint. However, there is a difference with the situation of Caire et al. (1999) in that for our problem the quantity $P_{k}$ is not memoryless and thus the sub-problem is not always feasible, so it may not always be possible to satisfy $P_{k+1}=D$ in a single time step for arbitrary $P_{k}$, depending on which of the conditions of Section 3 the distortion threshold $D$ satisfies.

For those $D$ 's satisfying the condition $D \geq D_{1}$ of Section 3 , the sub-problem is always feasible and this policy can be applied directly. For the condition $D_{2}<D<D_{1}$ of Section 3, if $P_{k}$ is such that $P_{k+1}=D$ cannot be achieved in one time step, one should arguably still transmit with some power (since not transmitting will actually cause the error covariance to increase even further) to reduce $P_{k+1}$ so that in future time steps, e.g. $P_{k+2}=D$ can then be achieved. The heuristic we propose in this case is to transmit with sum power equal to $\eta s^{*}$, using the allocation that minimizes $P_{k+1}$ subject to the constraint $\sum_{i=1}^{M} \gamma_{i, k}=\eta s^{*}$. Here $s^{*}$ is the power threshold and $\eta \leq 1$ is a constant to be chosen by us. From numerical simulations, we have found that values of $\eta$ around the range $1 / 5-1 / 20$ result in very good performance.

To summarise, the proposed suboptimal power allocation policy that covers both the situations $D \geq D_{1}$ and $D_{2}<$ $D<D_{1}$ is as follows:

- Let $P_{k}, g_{k}$ be given.

- If $P_{k+1}=D$ can be achieved for this value of $P_{k}$, solve the following problem:

$$
\begin{aligned}
& \min _{\alpha_{k}} \sum_{i=1}^{M} \gamma_{i, k}=\sum_{i=1}^{M} \alpha_{i, k}^{2}\left(\frac{c_{i}^{2} \sigma_{w}^{2}}{1-a^{2}}+\sigma_{i}^{2}\right) \\
& \text { s.t. } P_{k+1}=D
\end{aligned}
$$

- If the minimizing sum power is less than the threshold $s^{*}$, then transmit using this power allocation, and update the error covariance as $P_{k+1}=D$.

- Otherwise transmission is turned off, and update the error covariance as $P_{k+1}=a^{2} P_{k}+\sigma_{w}^{2}$.

- If $P_{k+1}=D$ cannot be achieved for this value of $P_{k}$, solve the following problem:

$$
\begin{aligned}
& \min _{\alpha_{k}} P_{k+1} \\
& \text { s.t. } \sum_{i=1}^{M} \alpha_{i, k}^{2}\left(\frac{c_{i}^{2} \sigma_{w}^{2}}{1-a^{2}}+\sigma_{i}^{2}\right)=\eta s^{*}
\end{aligned}
$$

Transmit using the power allocation provided by the solution to (14), and update the error covariance using (4).

The sub-problems (13) and (14) have previously been shown to be convex optimization problems, and furthermore can be solved analytically for any number of sensors, see Leong et al. (2008), also Cui et al. (2007), for further details.

\section{NUMERICAL RESULTS}

\subsection{Single sensor}

Consider first an example with $a=0.8, c_{1}=1, \sigma_{1}^{2}=1$, $\sigma_{w}^{2}=\sigma_{n}^{2}=1$. With these parameters the quantities $D_{1}$ and $D_{2}$ from Section 3 have values $D_{1}=1.4706, D_{2}=$ 1.3700. Also, $\sigma_{w}^{2} /\left(1-a^{2}\right)=2.7778$. The fading channel is assumed to be Rayleigh, with $g_{k}$ being exponentially distributed with mean 1 .

Figure 1 plots the outage probability and average power obtained from the MDP solution, for various $D$ values. We use 100 discretization points for each of the quantities $P_{k}$, $g_{k}, \gamma_{k}$. We discretize $P_{k}$ over the range $D_{2}$ to $\sigma_{w}^{2} /\left(1-a^{2}\right)$, and $g_{k}$ over the range 0 to 15 . The discretization range for the power $\gamma_{k}$ is from 0 to $\gamma_{\max }$, where $\gamma_{\max }$ varies for different average power/outage probability requirements. As a rule of thumb we took $\gamma_{\max }$ to be at least twice the maximum power $s^{*}$ used in the suboptimal policy, for a similar average power/outage probability trade-off. The relative value iteration algorithm is run for 20 iterations in solving (10) for each value of the weighting parameter $\beta$. We see from Figure 1 that smaller $D$ values require more power to be transmitted for a given outage probability.

We next compare the performance of the suboptimal policy with the MDP solution. Figure 2 plots the outage probability and average power obtained from the MDP solution and suboptimal policy, for $D=2.0$. Since $2.0>$ $1.4706=D_{1}$, this is the case where $P_{k+1}=D$ can always be achieved in one time step. Figure 3 plots the outage probability and average power obtained from the MDP solution and suboptimal policy, for $D=1.4$. In this case we have $D_{2} \leq D \leq D_{1}$, and we will use $\eta=1 / 5$ for the suboptimal policy. In both graphs, it can be seen that the suboptimal policy gives very close performance to the solution obtained by solving the MDP.

To shed some insight into why the suboptimal policy performs so well, in Figure 4 we plot for $D=2.0$ and 


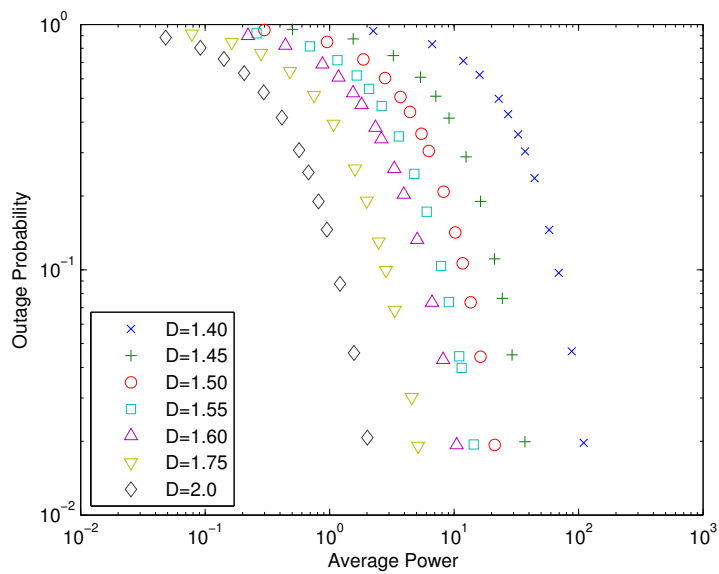

Fig. 1. Outage probability and average power for various $D$ values.

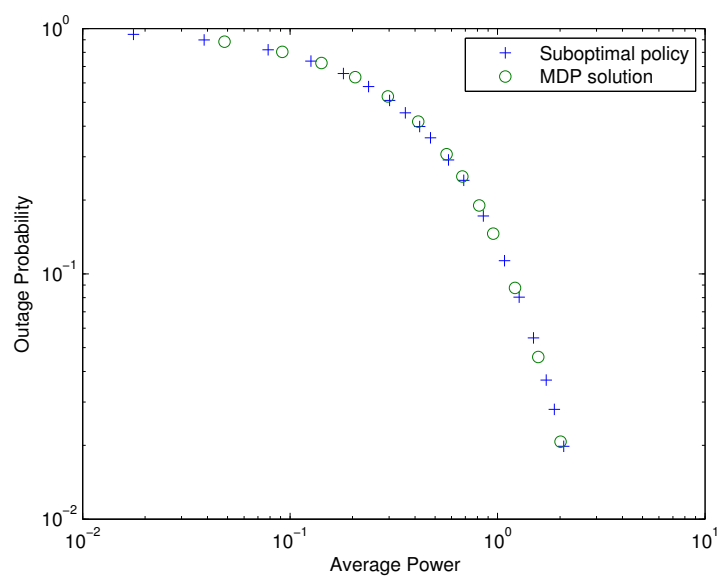

Fig. 2. Outage probability and average power for MDP and suboptimal policy, with $D=2.0$.

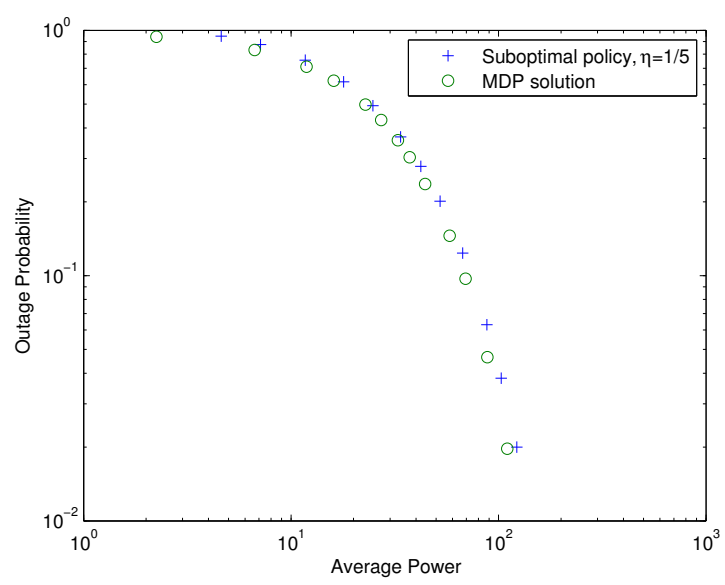

Fig. 3. Outage probability and average power for MDP and suboptimal policy using $\eta=1 / 5$, with $D=1.4$.

$P_{k}=2.28$, the power allocation obtained from solving the MDP as a function of $g_{k}$, together with the corresponding value of $P_{k+1}$ when using this power allocation. For values of $g_{k}$ less than around 5 , the power allocation is such that $P_{k+1}=D=2.0$ is met provided the power required is less
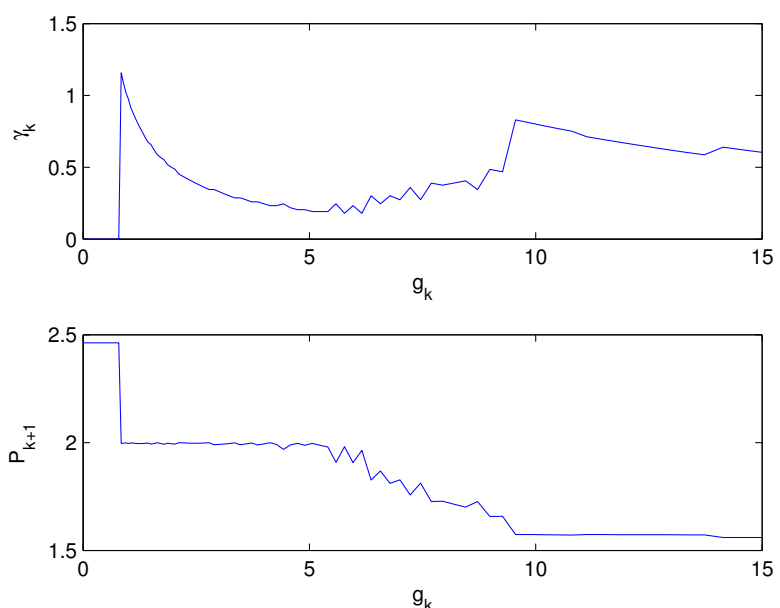

Fig. 4. Power allocations obtained from MDP solution, for a fixed $P_{k}$

than some threshold, which corresponds to the behaviour of the suboptimal policy. Since $P\left(g_{k}>5\right)=\exp (-5) \approx$ $6.74 \times 10^{-3}$ is quite small, we see that most of the time the MDP solution behaves like the suboptimal policy. For values of $g_{k}$ greater than 5 , the power allocated is more than that required to satisfy $P_{k+1}=D$, until around values of $g_{k}$ greater than 10, where the power allocated makes $P_{k+1} \approx 1.5625$. We notice that $a^{2} \times 1.5625+\sigma_{w}^{2}=$ 2.0 , so the value of $P_{k+1}=1.5625$ implies that $P_{k+2}=D$ will be achieved even without the sensor transmitting anything at time $k+1$. This qualitative behaviour in the power allocation functions obtained from the MDP solution can also be observed for other values of $P_{k}$.

\subsection{Multiple sensors}

We now consider a two sensor example with $a=0.8$, $c_{1}=1, c_{2}=1, \sigma_{1}^{2}=1, \sigma_{2}^{2}=2, \sigma_{w}^{2}=\sigma_{n}^{2}=1$. With these parameters the quantities $D_{1}$ and $D_{2}$ now have the values $D_{1}=1.3441, D_{2}=1.2806$. The fading channels for both sensors are assumed to be Rayleigh. Due to the increase in computational complexity, we now use 20 discretization points for each of the quantities $P_{k}, g_{1, k}, g_{2, k}, \gamma_{1, k}, \gamma_{2, k}$ here when solving the MDP. Figure 5 plots the outage probability and average sum power obtained from the MDP solution and suboptimal policy using $\eta=1 / 5$, for a distortion $D=1.3$. Again the two graphs are very close to each other.

We next consider the effect of increasing the number of sensors $M$. For simplicity we consider a "symmetric" situation with $a=0.8, \sigma_{w}^{2}=\sigma_{n}^{2}=1, c_{i}=1, i=1, \ldots, M, \sigma_{i}^{2}=$ $1, i=1, \ldots, M$. The fading channels are all taken to be Rayleigh, and we let the distortion threshold $D=1.5$. As solving the MDP is prohibitively expensive computationally for $M>2$, we will only present the results for the sub-optimal policy, which can be easily generated. Figure 6 plots the outage probability and average sum power for this situation, where we can readily see the outage performance improvements from using multiple sensors. 


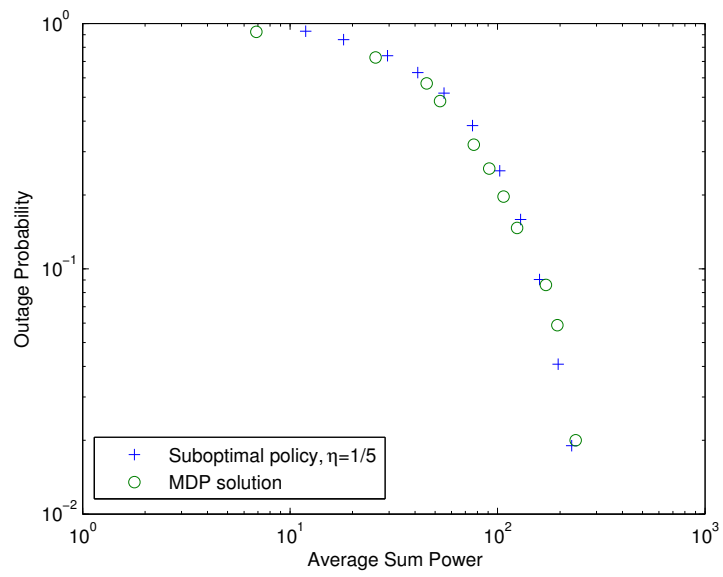

Fig. 5. Two sensor case. Outage probability and average sum power for MDP and suboptimal policy using $\eta=1 / 5$, with $D=1.3$.

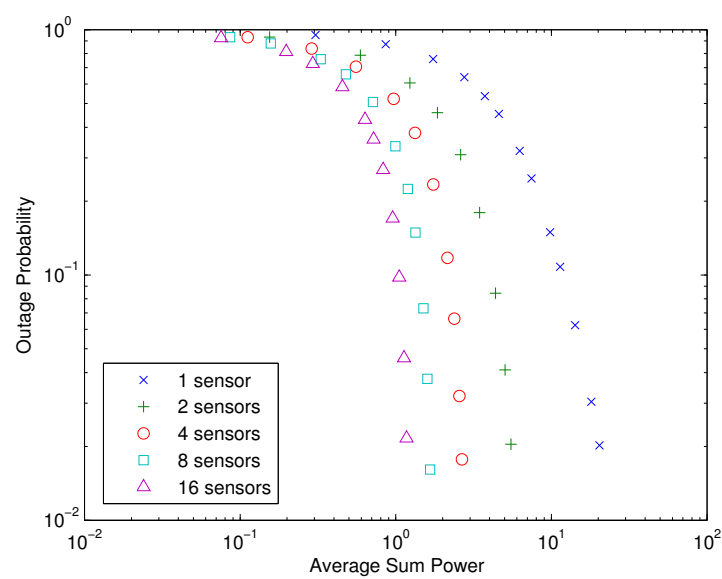

Fig. 6. Outage probability and average sum power for different numbers of sensors, using the sub-optimal policy

\section{UNSTABLE SYSTEMS}

In this section we will consider the outage minimization problem for unstable systems. Since for unstable systems meeting the outage constraints requires increasingly large amounts as power as the time increases, we will instead consider a finite horizon version of problem (6). ${ }^{2}$ We can write this as

$$
\begin{aligned}
& \min _{\left\{\gamma_{k}\right\}} \frac{1}{K} \sum_{k=0}^{K-1} \mathbb{E}\left[1\left(P_{k+1}>D\right)\right] \\
& \text { s.t. } \frac{1}{K} \sum_{k=0}^{K-1} \mathbb{E}\left[\sum_{i=1}^{M} \gamma_{i, k}\right] \leq \mathcal{P}
\end{aligned}
$$

where $K$ is the finite horizon over which we wish to solve the problem. The sensor transmit powers are as defined in (5), but for unstable systems $\mathbb{E}\left[x_{k}^{2}\right]$ is now time varying, and given by

$$
\mathbb{E}\left[x_{k}^{2}\right]=a^{2 k} P_{0}+\left(a^{2 k-2}+\cdots+a^{2}+1\right) \sigma_{w}^{2}
$$

2 Another possibility is to consider a discounted cost version of problem (6). with initial covariance $\mathbb{E}\left[x_{0}^{2}\right]=P_{0}$. Introducing the Lagrangian

$$
L^{\beta, K}=\frac{1}{K}\left\{\sum_{k=0}^{K-1} \mathbb{E}\left[1\left(P_{k+1}>D\right)\right]+\beta \sum_{k=0}^{K-1} \mathbb{E}\left[\sum_{i=1}^{M} \gamma_{i, k}\right]\right\},
$$

we now wish to solve the unconstrained problem

$$
\min _{\gamma_{k}} \frac{1}{K} \sum_{k=0}^{K-1} \mathbb{E}\left[l^{\beta}\left(P_{k}, g_{k}, \gamma_{k}\right) \mid P_{0}, g_{0}\right]
$$

where $l^{\beta}\left(P_{k}, g_{k}, \gamma_{k}\right) \equiv 1\left(P_{k+1}>D\right)+\beta \sum_{i=1}^{M} \gamma_{i, k}$. The discretized version of problem (16) may then be solved numerically using the standard dynamic programming algorithm. Numerical results for the finite horizon unstable case cannot be included here due to space limitations and will be reported elsewhere.

\section{CONCLUSION}

We have considered the outage minimization problem for state estimation of scalar linear systems. For stable systems we used an infinite horizon problem formulation and for unstable systems we used a finite horizon formulation. Extensions of this work to vector systems is currently under investigation.

\section{REFERENCES}

Bertsekas, D.P. (2000). Dynamic Programming and Optimal Control, Volumes I and II. Athena Scientific, Belmont, Massachusetts, 2nd edition.

Caire, G., Taricco, G., and Biglieri, E. (1999). Optimum power control over fading channels. IEEE Trans. Inform. Theory, 45(5), 1468-1489.

Cui, S., Xiao, J.J., Goldsmith, A., Luo, Z.Q., and Poor, H.V. (2007). Estimation diversity and energy efficiency in distributed sensing. IEEE Trans. Signal Processing, $55(9), 4683-4695$.

Gastpar, M. and Vetterli, M. (2003). Source-channel communication in sensor networks. Springer Lecture Notes in Computer Science, 2634, 162-177.

Ghasemi, N. and Dey, S. (2008). Power-efficient dynamic quantization for multisensor HMM state estimation over fading channels. In Proc. ISCCSP, 1553-1558. Malta.

Hanly, S.V. and Tse, D.N.C. (1998). Multiaccess fading channels - Part II: Delay-limited capacities. IEEE Trans. Inform. Theory, 44(7), 2816-2831.

Huang, M. and Dey, S. (2006). Dynamic quantizer design for hidden Markov state estimation via multiple sensors with fusion center feedback. IEEE Trans. Signal Processing, 54(8), 2887-2896.

Leong, A.S., Dey, S., and Evans, J.S. (2008). State estimation over wireless channels using multiple sensors: Asymptotic behaviour and optimal power allocation. URL http: //arxiv.org/abs/0803.3850.

Luo, J., Yates, R., and Spasojević, P. (2005). Service outage based power and rate allocation for parallel fading channels. IEEE Trans. Inform. Theory, 51(7), 2594-2611.

Negi, R. and Cioffi, J.M. (2002). Delay-constrained capacity with causal feedback. IEEE Trans. Inform. Theory, 48(9), 2478-2494.

Puterman, M.L. (1994). Markov Decision Processes: Discrete Stochastic Dynamic Programming. WileyInterscience, New York. 